\title{
The PPAR $\gamma 2$ P12A polymorphism is not associated with all-cause mortality in patients with type 2 diabetes mellitus
}

\author{
Antonio Pacilli ${ }^{1} \cdot$ Sabrina Prudente $^{2} \cdot$ Massimiliano Copetti $^{3} \cdot$ Andrea Fontana $^{3}$. \\ Luana Mercuri $^{2}$ - Simonetta Bacci ${ }^{1}$ - Antonella Marucci ${ }^{4} \cdot$ Federica Alberico $^{2}$. \\ Raffaella Viti ${ }^{1}$ - Antonio Palena ${ }^{1}$ - Olga Lamacchia ${ }^{5}$ - Mauro Cignarelli ${ }^{5}$. \\ Salvatore De Cosmo ${ }^{1} \cdot$ Vincenzo Trischitta $^{2,4,6}$
}

Received: 11 October 2015/ Accepted: 19 February 2016

(C) Springer Science+Business Media New York 2016

\begin{abstract}
The high mortality risk of patients with type 2 diabetes mellitus may well be explained by the several comorbidities and/or complications. Also the intrinsic genetic component predisposing to diabetes might have a role in shaping the risk of diabetes-related mortality. Among type 2 diabetes mellitus SNPs, rs 1801282 is of particular interest because (i) it is harbored by peroxisome proliferator-activated receptor- $\gamma 2(P P A R \gamma 2)$, which is the target for thiazolidinediones which are used as antidiabetic drugs, decreasing all-cause mortality in type 2 diabetes mellitus, and (ii) it is associated with insulin resistance and related traits, risk factors for overall mortality in type 2 diabetes mellitus. We investigated the role of $P P A R \gamma 2$ P12A, according to a dominant model (PA + AA vs. PP individuals) on incident all-cause mortality in three cohorts of type 2 diabetes mellitus, comprising a total of 1672
\end{abstract}

Vincenzo Trischitta

vincenzo.trischitta@uniroma1.it

1 Department of Medical Sciences, Scientific Institute "Casa Sollievo della Sofferenza”, San Giovanni Rotondo, FG, Italy

2 Mendel-Laboratory, Scientific Institute "Casa Sollievo della Sofferenza", San Giovanni Rotondo, FG, Italy

3 Unit of Biostatistics, Scientific Institute "Casa Sollievo della Sofferenza", San Giovanni Rotondo, FG, Italy

4 Research Unit of Diabetes and Endocrine Diseases, Scientific Institute "Casa Sollievo della Sofferenza", Viale Padre Pio, 71013 San Giovanni Rotondo, FG, Italy

5 Unit of Endocrinology and Metabolic Diseases, Department of Surgical and Medical Sciences, University of Foggia, Foggia, Italy

6 Department of Experimental Medicine, "Sapienza" University of Rome, Rome, Italy patients (462 deaths) and then performed a meta-analysis of ours and all available published data. In the three cohorts pooled and analyzed together, no association between $P P A R \gamma 2 \mathrm{P} 12 \mathrm{~A}$ and all-cause mortality was observed (HR $1.02,95 \%$ CI 0.79-1.33). Similar results were observed after adjusting for age, sex, smoking habits, and BMI (HR $1.09,95 \%$ CI $0.83-1.43$ ). In a meta-analysis of ours and all studies previously published $(n=3241$ individuals; 666 events), no association was observed between PPAR 2 P12A and all-cause mortality (HR 1.07, $95 \%$ CI $0.85-1.33)$. Results from our individual samples as well as from our meta-analysis suggest that the PPAR $22 \mathrm{P} 12 \mathrm{~A}$ does not significantly affect all-cause mortality in patients with type 2 diabetes mellitus.

Keywords Type 2 diabetes - PPAR 2 P12A SNP - Risk of death · Overall mortality

\section{Introduction}

The rate of mortality of patients with type 2 diabetes mellitus (T2DM) is twice as much as that of non-diabetic individuals of similar age [1]; this makes diabetes a leading risk factor for all-cause mortality, accounting for 4.9 million deaths worldwide [2]. Such high risk may well be explained by the several comorbidities and/or chronic complications which characterize diabetic patients, including abdominal obesity, hypertension, dyslipidemia, chronic renal dysfunction, and cardiovascular disease [1, 2]. Beyond these well-established risk factors, it could be hypothesized that also the intrinsic genetic component predisposing to diabetes itself has a role in shaping the risk of diabetes-related mortality. Such an intriguing hypothesis is compatible with the observation that some single 
nucleotide polymorphisms (SNPs), pointed by genomewide association studies (GWAS) as important risk factors of T2DM, have also been associated with mortality rate, among diabetic patients [3] as well as non-diabetic individuals [4].

Among the many so far established T2DM SNPs, rs1801282 [5] appears to be the most interesting one in the context of diabetes-related mortality because of several reasons: (i) it is harbored by peroxisome proliferatoractivated receptor- $\gamma 2$ (PPAR $\gamma 2)$ which encodes for a ligand-activated transcription factor involved in adipose tissue biology as well as lipid and glucose metabolism [6] and which, most importantly, is the target for thiazolidinediones, commonly used antidiabetic drugs able to reduce all-cause mortality in patients with T2DM [7]; (ii) it is one of the few non-synonymous T2DM SNPs (i.e., causing a proline to alanine amino acid substitution at codon 12; P12A) with a reported biological function as indicated by in vitro experiments [5]; (iii) it has been associated with insulin resistance [8], body mass index [9], high blood pressure [10], myocardial infarction [11, 12], and diabetic nephropathy [13-16], all well-established risk factors for overall mortality in patients with T2DM. Despite this strong background, only two studies $[12,17]$ have specifically investigated the role of $P P A R \gamma 2$ P12A SNP on all-cause mortality in T2DM, with conflicting results.

In order to get deeper insights about this issue, we investigated the role of $P P A R \gamma 2 \mathrm{P} 12 \mathrm{~A}$ on incident all-cause mortality in three cohorts of T2DM, comprising a total of 1672 Italian patients from a very homogeneous geographical area (i.e., Northern Apulia, in Central-Southern Italy), whose demographic and clinical features are carefully characterized [18, 19]. Subsequently, a meta-analysis on ours plus all available published data was carried out.

\section{Materials and methods}

\section{Ethics statement}

The study and the informed consent procedures were approved by the local Institutional Ethic Committee IRCCS (Istituto di Ricovero e Cura a Carattere Scientifico) "Casa Sollievo della Sofferenza" and performed according to the Helsinki Declaration. All participants gave written consent.

\section{Patients}

We studied three previously described [18, 19] cohorts of patients with T2DM (according to ADA 2003 criteria), followed up for all-cause mortality.

\section{The Gargano Mortality Study (GMS)}

One thousand twenty-eight Whites from Italy with T2DM were consecutively recruited at Scientific Institute "Casa Sollievo della Sofferenza" in San Giovanni Rotondo (Apulia, Central-Southern Italy) for a study aimed at unraveling predictors of incident all-cause mortality. The only exclusion criterion was the presence of poor life expectancy due to malignancies. Up to date, this cohort has been followed up for $10.7 \pm 3.6$ years (range: 0.1-14.1), with the last information on vital status being obtained on November 30, 2014. After excluding patients (i) whose information on vital status at follow-up was not available $(n=9)$, (ii) who had missing information on PPAR 2 P12A genotype at baseline $(n=103)$, and (iii) who were also part of the Gargano Heart Study $(n=116)$ (see below), 800 patients ( $77.8 \%$ of the initial cohort) constituted the eligible sample for the present investigation.

\section{The Gargano Heart Study (GHS)}

Three hundred forty Whites from Italy with T2DM and coronary heart disease, as indicated by previous myocardial infarction or $50 \%$ stenosis of at least one major vessel at coronary angiography, or both, were consecutively recruited at Scientific Institute "Casa Sollievo della Sofferenza" in San Giovanni Rotondo from 2000 to 2008. The only exclusion criterion was the presence of poor life expectancy due to malignancies. Up to date, this cohort has been followed up for $5.5 \pm 2.5$ years (range: 0.1-9.9), with the last information on vital status being obtained on March 31,2011 . After excluding patients (i) whose information on vital status at follow-up was not available $(n=23)$ and (ii) who had missing information on PPAR 2 P12A genotype at baseline $(n=39), 278$ patients $(81.8 \%$ of the initial cohort) constituted the eligible sample for the present investigation.

\section{The Foggia Mortality Study (FMS)}

One thousand one hundred two Whites from Italy with T2DM were consecutively recruited at Endocrine Unit of University of Foggia (Apulia, Central-Southern Italy) from January 7, 2002 to September 30, 2008, for a study aimed at unraveling predictors of incident all-cause mortality. Also in this case, the only exclusion criterion was the presence of poor life expectancy due to malignancies. Up to date, this cohort has been followed up for $7.1 \pm 1.4$ years (range: $4.4-11.7$ ), with the last information on vital status being obtained on March 31, 2014. After excluding patients (i) whose information on vital status at follow-up was not available $(n=101)$ and (ii) from whom DNA was not obtained $(n=407), 594$ patients $(53.9 \%$ of 
the initial cohort) constituted the eligible sample for the present analysis.

\section{Data collection}

At baseline, all patients were interviewed regarding age at diabetes diagnosis, smoking habits, and ongoing anti-diabetes, anti-dyslipidemia, and anti-hypertension treatments. Duration of diabetes was calculated from the calendar year of data collection minus the calendar year of diabetes diagnosis. All subjects enrolled in the study underwent physical examination, including measurements of height, weight, body mass index (BMI; $\mathrm{Kg} / \mathrm{m}^{2}$ ), and blood pressure (two measurements rounded to the nearest $2 \mathrm{mmHg}$ in the sitting position after at least 5 min rest, using an appropriate-sized cuff; diastolic blood pressure was recorded at the disappearance of Korotokoff sound, phase V). Fasting venous blood was sampled from an antecubital vein from all patients for the measurement of total serum cholesterol (enzymatic method, Cobas; Roche Diagnostics, Welwin Garden City, U.K.), HDL-cholesterol, serum triglycerides (enzymatic method, Cobas), and $\mathrm{HbA}_{1 \mathrm{c}}$ (HPLC Diamat Analyzer; Bio-Rad, Richmond, CA); LDLcholesterol was then calculated by the Friedewald formula.

\section{Genotyping}

Genomic DNA was extracted from peripheral blood according to standard procedures. The PPAR 2 P12A SNP (rs1801282) was genotyped by a TaqMan Pre-Designed SNP genotyping assay (C_1129864_10, Applied Biosystems, Foster City, CA) on ABI 7900HT genetic analyzer.

Genotyping quality was tested by including six blinded duplicate samples in each 96-well assay. The average agreement rate of duplicate samples was greater than $99 \%$.

\section{Study endpoint}

All-cause mortality was the only predetermined end point of this study. At follow-up, the vital status of study patients was ascertained by two authors for each study, either by telephone interview with the patient or his/her relatives or by queries to the registry office of cities of residence. For GMS, the last follow-up was carried out by queries to the Italian Health Card (http://sistemats1.sanita.finanze.it/wps/ portal/portalets/cittadinots/ts).

\section{Search strategy and selection criteria}

In order to perform a meta-analysis comprising data from our three study samples and those from published studies, two investigators (AP and SDC) independently searched PubMed and Web of Science for prospective studies with
PPAR 2 P12A as exposure and all-cause mortality as outcome, published until July 2015. Search terms used were $(P P A R \gamma 2$ Pro12Ala or PPAR 2 P12A or PPAR $\gamma N P$ or peroxisome proliferator-activated receptor gamma 2 Pro12Ala or peroxisome proliferator-activated receptor gamma $2 \mathrm{P} 12 \mathrm{~A}$ or peroxisome proliferator-activated receptor gamma $2 \mathrm{SNP}$ or rs1801282) and (mortality or death rate). Exclusion criteria were i) non-original papers; ii) study population other than type 2 diabetes; iii) different outcomes. The quality of the eligible studies was assessed by availability of data on both clinical features and distribution of allele/genotype and by available information on HWE and genetic model utilized, as well. SDC and AP extracted the data from such studies. No disagreements were observed.

\section{Statistical analysis}

Patients' baseline characteristics were reported as mean \pm standard deviation (SD) and frequency (percentage) for continuous and categorical variables, respectively.

The exact test for Hardy-Weinberg equilibrium (HWE) was carried out as previously described [20].

Overall age- and sex-adjusted mortality rates were assessed using Poisson model and expressed as number of deaths per 100 person-years. The overall survival was defined as the time between enrollment and death; for subjects who did not experience the end point, survival time was censored at the time of the last available followup visit. Time-to-death analyses were performed using univariable and multivariable Cox proportional hazards regression models and risks were reported as hazard ratios (HR) along with their $95 \%$ confidence intervals (CI). To test the effect of P12A genotype on all-cause mortality, due to the very low frequency of AA genotypes, HRs were reported for the presence of $\mathrm{PA}+\mathrm{AA}$ (named as XA) vs. PP genotypes, according to a dominant model of inheritance.

Pooled data meta-analysis was performed using a linear mixed-effect modeling and according to a dominant model of inheritance because only the latter genetic model was reported in the previous published studies selected for our meta-analysis.

Statistical heterogeneity among studies was assessed using the Cochran Q-test and heterogeneity hold for $p$ values less than 0.10 [21]. Study-specific estimates were pooled using either the fixed-effects model or, in presence of heterogeneity (i.e., Q-test statistically significant), the random effects model [22]. Following Sterne et al. [23] and Ioannidis et al. [24] recommendations, the publication bias was evaluated by the visual inspection of a funnel plot (with a $95 \%$ pseudo-CI region) without performing any statistical test, due to the small number of studies (fewer 
than ten). For forest plot, a square was plotted for each study whose center projection on the underlying scale corresponds to the study-specific HR. The area of the square was proportional to the inverse of the variance of the logarithm transformation of HR (i.e., precision of the HR estimates) and thus gives a measure of the amount of statistical information available from that particular estimate. A diamond was used to plot the summary HRs, the center of which represents the HR; the extremes of the summary HRs show the $95 \%$ CI.

A $p$ value $<0.05$ was considered to be statistically significant. All analyses were performed using SAS Release 9.3 (SAS Institute, Cary, NC, USA) and R 2.15 (package: metafor).

\section{Sample size calculation}

A sample of 1672 subjects (GMS, GHS, and FMS) achieves $80 \%$ (or $90 \%$ ) power, assuming a Type I Error of $5 \%$, to detect an HR of 1.39 (or 1.45) assuming a dominant model of inheritance (see above) and an anticipated event rate of 0.28 , the average value of our three samples (see below).

\section{Results}

\section{Present studies}

Baseline clinical features of the 1672 T2DM patients from the three study samples are reported in Table 1. Minor allele (i.e. A12) frequency was similar in the three samples (Table 1). Clinical features were not different across P12A genotypes in any study sample (data not shown).

In GMS (follow-up $=10.7$ years), GHS (followup $=5.5$ years), and FMS (follow-up $=7.1$ years), 246 (30.7\%), $64(23.0 \%)$, and $152(25.6 \%)$ patients died, with an age- and sex-adjusted annual mortality rate of 2.2 , 3.8 , and 2.5 per 100 person-years, respectively.

Genotype distribution did not depart from the HWE in GMS and FMS ( $p$ values: 0.419 and 0.351 , respectively) and minimally did so in GHS ( $p=0.023$ ).

Given the very low number of patients homozygous for the A allele, data from such individuals were pooled together with those from PA patients, considered as a single group and named as XA individuals.

Mortality incidence rates in each cohort across genotype groups are reported in Table 2, while Table 3 shows the HRs $(95 \% \mathrm{CI})$ for all-cause mortality, following a dominant genetic model of the P12A genotypes.

Given the absence of heterogeneity in the association with all-cause mortality (i.e. $p$ for study sample-by-SNP interaction $=0.56$ ), the three cohorts were pooled and analyzed together, so to increase statistical power. In a model where only "study sample" was used as adjusting covariate, no significant association between PPAR 2 P12A and all-cause mortality was observed (Table 3). Similar results were observed when also age, sex, smoking habits, and BMI were added into the model.

In post hoc analyses on pooled data, no effect on the association between the P12A SNP and all-cause mortality was observed after stratifying individuals according to either BMI status $\left(<30 \mathrm{~kg} / \mathrm{m}^{2}, n=803 ; \geq 30 \mathrm{~kg} / \mathrm{m}^{2}\right.$, $n=810$; BMI data were missing for 59 individuals; $p$ for SNP-by-BMI status interaction $=0.25$ ) or sex (male, $n=862 ; \quad$ female, $n=810 ; \quad \mathrm{p}$ for SNP-by-sex interaction $=0.46$ ).

In addition, in the GHS, where data on death of cardiovascular origin were also available, no association between PPAR 2 P12A and cardiovascular mortality was observed $(p=0.48$, following a dominant genetic model of inheritance).

\section{Meta-analysis on present and previous studies}

Eighteen papers passed the filter of our inclusion criteria. Of those, 15 papers were excluded because they were (i) reviews rather than original papers $(n=2)$; (ii) investigating individuals from sets other than T2DM, including either the general population $(n=6)$, or type 1 diabetes mellitus $(n=1)$, or coronary artery disease $(n=2)$, or end stage renal disease $(n=1)$, or lung cancer disease $(n=1)$; (iii) with different study designs either for the exposure variable (i.e., variability of the $P P A R \alpha$, rather than $P P A R \gamma 2$, gene, $n=1$ ) or the outcome of interest (i.e., renal dysfunction, rather than all-cause mortality, $n=1$ ). At the end of this process, two studies [12,17] were considered eligible for our meta-analysis (Fig. 1). In the Go-DARTS, 2016 Whites with T2DM were enrolled; mean age and duration of diabetes were 64.4 and 7.9 years, respectively. No other clinical information is available about this sample. In the Szeto's study, all 220 subjects had T2DM and diabetic nephropathy with GFR $<60 \mathrm{ml} / \mathrm{min}$; mean age and duration of diabetes were 65.2 and 6.6 years in pre-dialysis patients, 60.9 and 7.9 years in dialysis patients; mean BMI was $22.0 \mathrm{~kg} / \mathrm{m}^{2}$ in pre-dialysis patients and $22.4 \mathrm{~kg} / \mathrm{m}^{2}$ in dialysis patients; systolic and diastolic blood pressure were 144.7 and $80.9,151.0$, and $82.1 \mathrm{mmHg}$ in pre-dialysis and dialysis patients, respectively. Genotyping for PPAR 2 Pro12Ala was performed using Taqman (Applied Biosystem) allelic discrimination assays in the Go-DARTS and PCR-RFLP analysis in Szeto's study; both samples were in HWE. In both studies included in the meta-analysis, the multivariable model (Cox hazards model) comprised age, sex, cholesterol, and blood pressure; in addition, Doney's 
Table 1 Baseline clinical features of study patients with type 2 diabetes mellitus

\begin{tabular}{llll}
\hline & GMS $n=800$ & GHS $n=278$ & FMS $n=594$ \\
\hline Sex (M/F) & $389 / 411$ & $183 / 95$ & $290 / 304$ \\
Age (years) & $61.6 \pm 9.8$ & $64.6 \pm 8.0$ & $63.1 \pm 11.9$ \\
Body mass index $\left(\mathrm{kg} / \mathrm{m}^{2}\right)$ & $30.1 \pm 5.8$ & $30.4 \pm 5.0$ & $30.1 \pm 6.2$ \\
Smokers: n (\%) & $109(13.6)$ & $48(18.6)$ & $90(16.5)$ \\
Duration of diabetes (years) & $10.4 \pm 8.7$ & $14.5 \pm 9.5$ & $13.3 \pm 10$ \\
Glycated hemoglobin (\%) & $8.7 \pm 2.0$ & $8.7 \pm 1.9$ & $8.9 \pm 2.2$ \\
Systolic blood pressure (mmHg) & $135.1 \pm 16.4$ & $135.2 \pm 20.0$ & $130.6 \pm 15.2$ \\
Diastolic blood pressure (mmHg) & $78.8 \pm 8.8$ & $76.2 \pm 10.0$ & $76.5 \pm 9.0$ \\
Low density lipoprotein cholesterol (mg/dl) & $121.3 \pm 38.0$ & $102.9 \pm 39.5$ & $100.8 \pm 37.1$ \\
Antidiabetic therapy & & & \\
Diet alone: $n$ (\%) & $110(14.2)$ & $17(6.4)$ & $73(13)$ \\
Oral antidiabetic drugs: $n(\%)$ & $344(44.6)$ & $90(34.1)$ & $272(48.5)$ \\
Insulin \pm oral antidiabetic drugs: $n(\%)$ & $318(41.2)$ & $157(59.5)$ & $213(38.5)$ \\
Anti-hypertensive therapy: $n(\%)$ & $389(53.2)$ & $235(85.8)$ & $395(70.3)$ \\
Anti-dyslipidemic therapy: $n(\%)$ & $233(29.3)$ & $173(64.3)$ & $211(37.6)$ \\
Number of patients with PP/PA/AA genotype & $691 / 107 / 2$ & $238 / 35 / 5$ & $509 / 84 / 1$ \\
MAF (\%) & 6.9 & 8.1 & 7.2 \\
\hline
\end{tabular}

Data are expressed as mean \pm standard deviation (SD) or frequency (percentage)

GMS Gargano Mortality Study, GHS Gargano Heart Study, FMS Foggia Mortality Study, PP patients homozygous for the $\mathrm{P}$ variant, $P A$ heterozygous patients, $A A$ patients homozygous for the A variant. MAF minor allele frequency (A12 in our case)
Table 2 Mortality incidence rates, along with $95 \%$ confidence intervals, in PPAR 2 PP and XA (i.e., PA/AA) genotypes

\begin{tabular}{lll}
\hline & PP & XA \\
\hline GMS & $2.9(95 \%$ CI $2.5-3.3)$ & $2.8(95 \%$ CI $2.0-4.0)$ \\
GHS & $4.3(95 \%$ CI 3.3-5.6) & $3.4(95 \%$ CI $1.7-6.7)$ \\
FMS & $3.5(95 \%$ CI 2.9-4.1) & $4.3(95 \%$ CI 3.0-6.3) \\
\hline
\end{tabular}

Incidence rates are expressed as number of death events per 100 person-years, along with their $95 \%$ confidence interval (95\% CI)

GMS Gargano Mortality Study, GHS Gargano Heart Study, FMS Foggia Mortality Study, PP patients homozygous for the $\mathrm{P}$ variant, $X A$ heterozygous patients or patients homozygous for the A variant

study also included in the model duration of diabetes, triglycerides, and BMI; Szeto's study also included HbA1c, GFR, proteinuria, CRP, and Charlson's comorbidity score.

We then performed an aggregate data meta-analysis comprising data from our three study samples and those from Doney et al. [12] and from Szeto et al. [17] (Q-test $p$ for heterogeneity $=0.19$ ), for a total of 3241 individuals and 666 events. As clearly shown in Fig. 2, no significant association was observed between PPAR 2 P12A SNP (XA vs. PP genotypes) and all-cause mortality (HR 1.07, $95 \%$ CI $0.85-1.33, p=0.57)$. Moreover, no publication bias was detected by the visual inspection of the funnel plot (Fig. 3).

\section{Discussion}

The established role of T2DM in increasing mortality risk $[1,2]$ makes reasonable testing the association between genetic determinants of T2DM and all-cause mortality rate $[3,4]$. In the specific context of the PPAR $\gamma 2$ P12A SNP, which has been reported to shape the risk not only of T2DM but also of insulin resistance [8], body mass index [9], high blood pressure [10], myocardial infarction [11, 12], and diabetic nephropathy [13-16], it is conceivable to hypothesize a reduced mortality risk among T2DM subjects carrying the A allele. However, the data we here present on a total of 3241 individuals clearly indicates a neutral effect of the PPAR $\gamma 2 \mathrm{P} 12 \mathrm{~A}$ SNP on all-cause mortality in T2DM.

In addition, neither BMI nor gender, which have been reported to influence the association between the $\mathrm{P} 12 \mathrm{~A}$ SNP and T2DM [25], cardiovascular events [26], human longevity [27, 28], and adiposity [29], played a role as modifiers of the P12A SNP effect on mortality rate.

No association between this SNP and all-cause mortality was observed not only in each of our three study samples, but also in patients from the large Go-DARTS [12], making thus not surprising that no association at all was observed in the meta-analysis of all so far available studies, comprising a total of 3241 individuals and 666 events. In all, although the small Szeto's study reported a significant 
Table 3 Hazard ratios, along with $95 \%$ confidence intervals, of all-cause mortality for PPAR 2 PA/AA versus PP genotypes

\begin{tabular}{|c|c|c|c|c|}
\hline & Model adjustments & HR & $95 \% \mathrm{CI}$ & $p$ value \\
\hline \multirow[t]{2}{*}{ GMS } & Unadjusted & 0.97 & $0.67-1.41$ & 0.87 \\
\hline & Adjusted for age, sex, smoking habits, and body mass index & 1.16 & $0.80-1.69$ & 0.44 \\
\hline \multirow[t]{2}{*}{ GHS } & Unadjusted & 0.79 & $0.38-1.66$ & 0.54 \\
\hline & Adjusted for age, sex, smoking habits, and body mass index & 1.10 & $0.49-2.48$ & 0.82 \\
\hline \multirow[t]{2}{*}{ FMS } & Unadjusted & 1.01 & $0.67-1.55$ & 0.95 \\
\hline & Adjusted for age, sex, smoking habits, and body mass index & 0.80 & $0.51-1.27$ & 0.34 \\
\hline \multirow[t]{2}{*}{ Whole sample } & Adjusted for study sample & 1.02 & $0.79-1.33$ & 0.86 \\
\hline & Adjusted for study sample, age, sex, smoking habits, and body mass index & 1.09 & $0.83-1.43$ & 0.53 \\
\hline
\end{tabular}

GMS Gargano Mortality Study; GHS Gargano Heart Study, FMS Foggia Mortality Study

Results are reported as Hazard Ratios (HR), along with their $95 \%$ confidence interval (95\% CI). Outcome: all-cause mortality

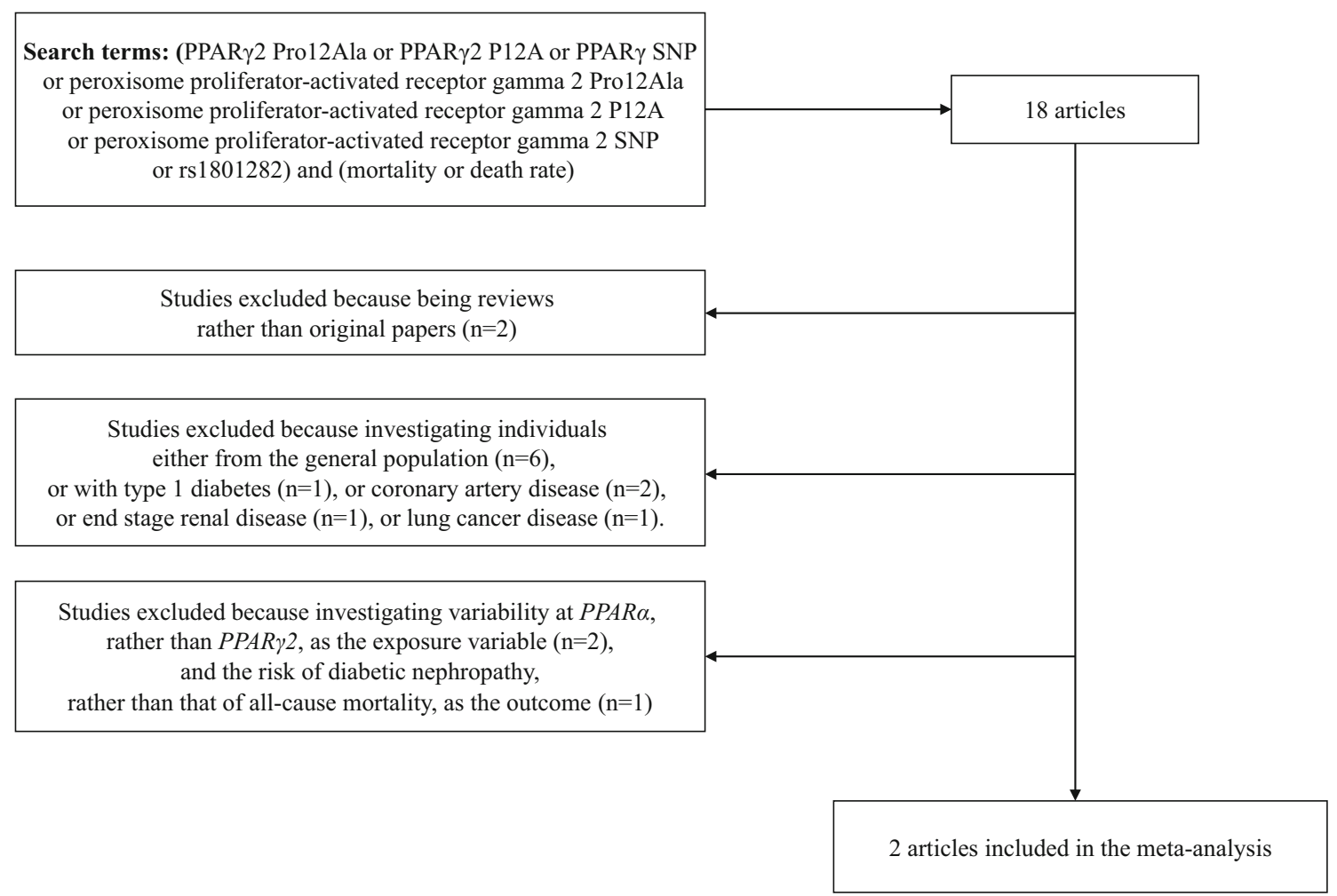

Fig. 1 Search strategy for selecting studies to include in meta-analysis for the association between PPAR 2 P12A SNP and all-cause mortality risk (search last run on July 2015)

association, a minimal and definitively not significant heterogeneity across all results was observed, thus minimizing the risk that the lack of some information from the two previous studies has flawed our meta-analysis. Our negative data are in contrast with those of two GWASderived genetic variations associated with T2DM, including that at 9p21 chromosome (i.e., rs10811661, near $C D K N 2 A / C D K N 2 B$ ) and that at the TCF7L2 locus (i.e., rs7903146) for which, in fact, an association with all-cause mortality was reported either in diabetic [3] or in nondiabetic [4] individuals. Such reported associations are somehow surprising, given that the two latter SNPs do not play any role on insulin resistance; rather, they affect insulin secretion [30], which is not known to affect mortality risk. So, present and previous [3, 4] data on the role of GWAS-derived SNPs for T2DM on mortality rate suggest that those of them which modulate the risk of death rather than doing so through their deleterious effect on 


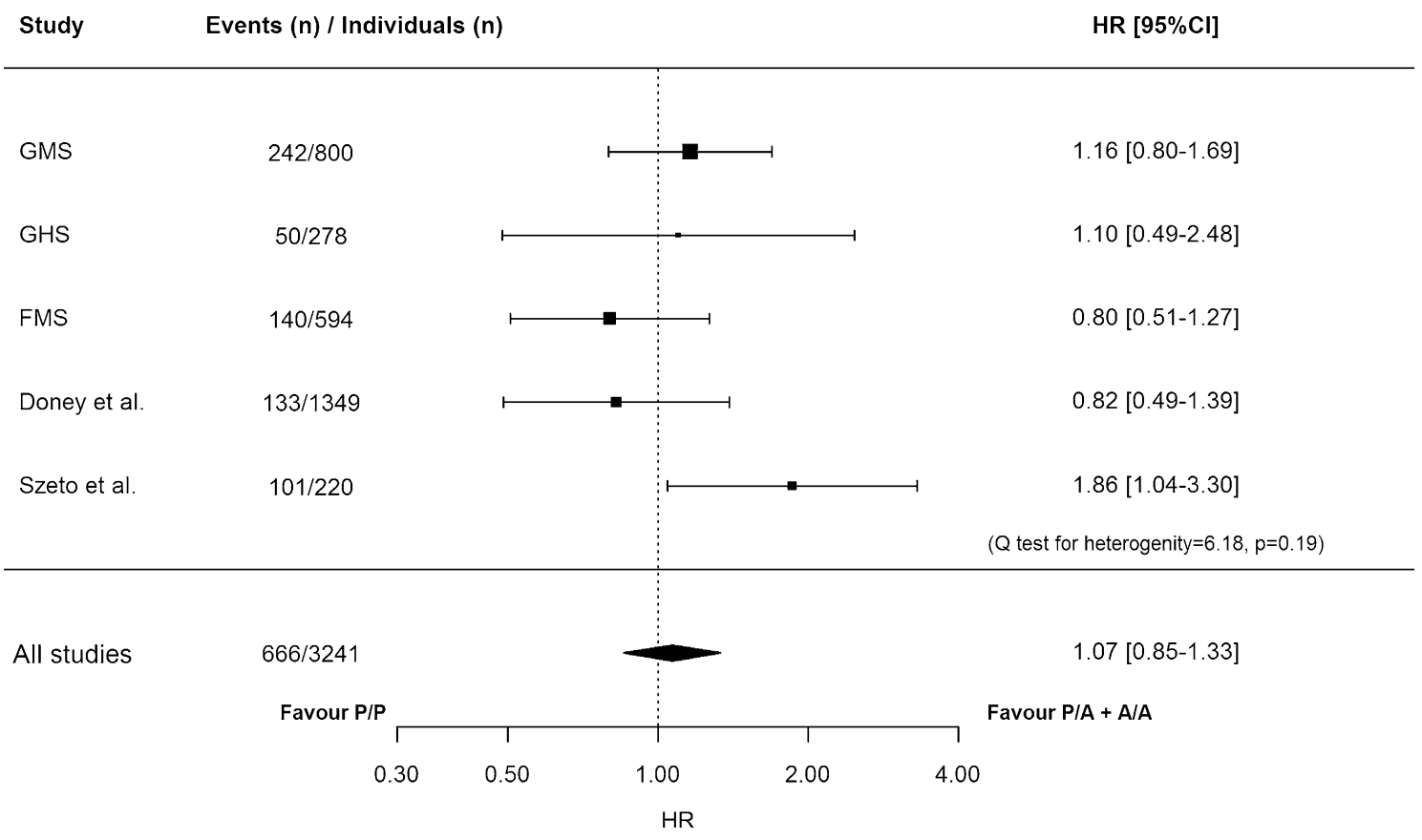

Fig. 2 Forest plot for fixed-effects meta-analysis for the association between PPAR 2 P12A SNP (following a dominant model of inheritance) and all-cause mortality risk. GMS Gargano Mortality Study, GHS Gargano Heart Study, FMS Foggia Mortality Study. Results are reported as Hazard Ratios (HR), along with their $95 \%$ confidence intervals (95\% CI). Model adjustments: GMS, GHS, and FMS: data are adjusted for age, sex, smoking habits, and body mass index. Doney et al.: data are provided as adjusted for age, sex, and smoking habits. Szeto et al.: data are provided as unadjusted
Fig. 3 Funnel plot for fixedeffects meta-analysis for the association between PPAR 2 P12A (following a dominant model of inheritance) and allcause mortality. GMS Gargano Mortality Study, GHS Gargano Heart Study, FMS Foggia Mortality Study. HR Hazard Ratios. Vertical reference line was drawn to intercept the $\mathrm{X}$-axis at the pooled $\mathrm{HR}$ estimate

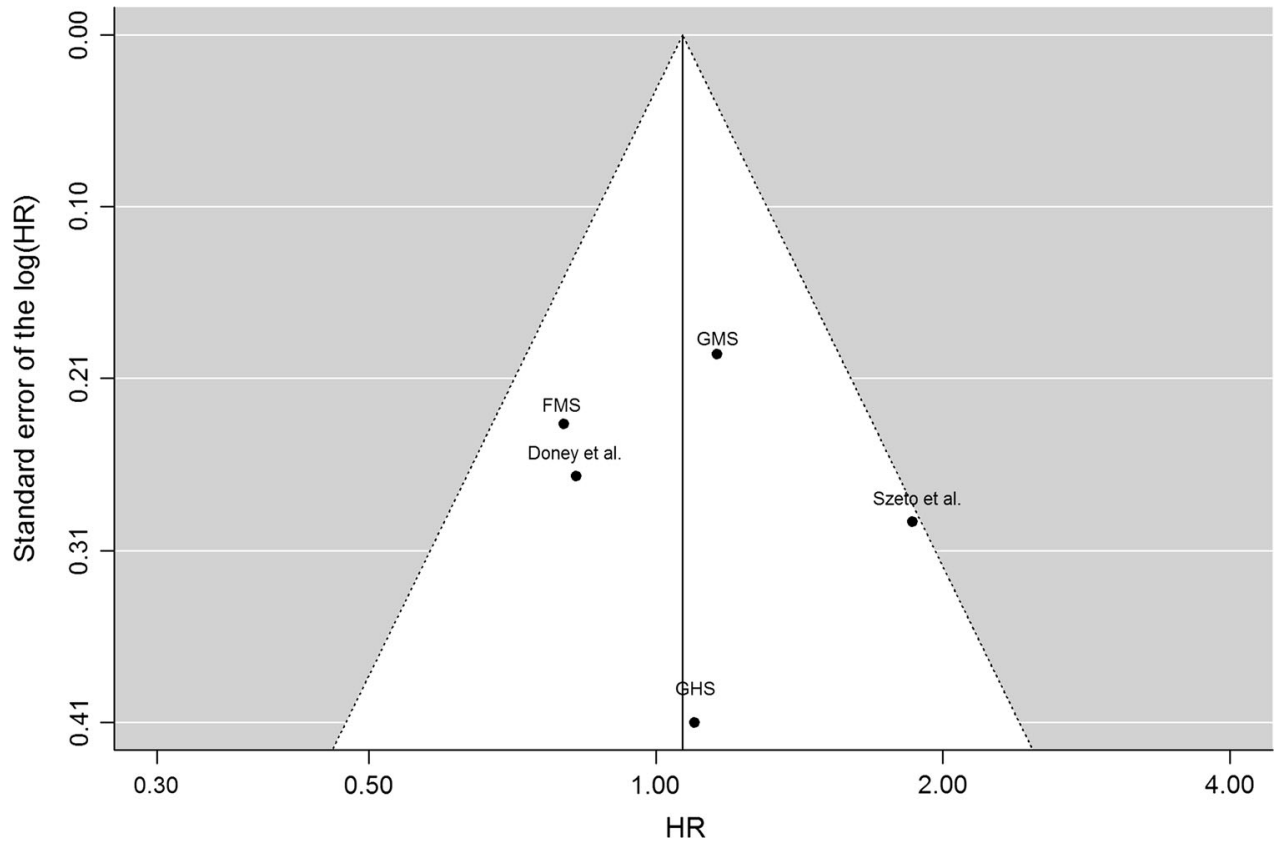

glucose homeostasis are likely to act through a yet unraveled, pleiotropic effect on different pathways.

We recently reported a combined effect of insulin signaling genes, which contribute to both insulin resistance [31] and T2DM [32] on all-cause mortality [33], a finding which is fully compatible with the well-known deleterious role of insulin resistance on risk of mortality rate [34, 35]. Previous [33] and present data, taken together, are compatible with the hypothesis that not all forms of insulin resistance are deleterious for life expectancy with only those based on altered insulin signaling, but not those presumably based on abnormal adipose tissue biology, being really able to increase the risk of death. 
The strength of our study is that the three samples here analyzed are quite homogenous in terms of clinical features and both environmental and genetic backgrounds, the two Institutions in which recruitment was carried out being only 50 kilometers apart. As a matter of fact, these samples have been very useful in our hands in addressing the role on mortality rate in T2DM of several genetic $[33,36]$ and non-genetic risk factors [37-42].

It is worth to underline that, given the geographical homogeneity of the population studied, the effect of population stratification was not explored and that, following Sterne et al. [23] and Ioannidis et al. [24] recommendations, we did not perform tests for publication bias, due to the small number of studies meta-analyzed. Also because of this, no study-specific results were assessed.

Our data have to be taken with caution, keeping in mind that we cannot exclude that in T2DM the P12A SNP is associated with less than $40 \%$ increased risk of mortality rate, a cut-off below which we do not have enough statistical power to draw firm conclusions. We call for caution also because while the three largest studies so far carried out in different clinical sets have obtained similar negative data [12, 43, 44], some smaller studies have reported positive, though conflicting, data, including increasing [45] or decreasing [46] all-cause mortality rate in homozygous individuals for the A12 variant or increasing of such rate [47] in homozygous individuals for the P12 variant.

Finally, in two of our three study cohorts we had no information on cardiovascular mortality, thus making it impossible to robustly investigate the role of P12A SNP on it, which has been previously described in normoglycemic males with symptomatic coronary artery disease at baseline and on $40 \mathrm{mg} /$ day pravastatin treatment [48].

In conclusion, results from our individual samples as well as from our meta-analysis of all available studies suggest that the PPAR 2 P12A SNP does not significantly affect all-cause mortality in patients with T2DM.

Funding This study was supported by the Italian Ministry of Health (Ricerca Corrente 2014 and 2015 to S.P., S.D.C. and V.T. and Ricerca Finalizzata 2009 to S.D.C.).

\section{Compliance with ethical standards}

Conflict of interest The authors declare that they have no conflict of interest.

\section{References}

1. Emerging Risk Factors Collaboration, S.R. Seshasai, S. Kaptoge, A. Thompson, E. Di Angelantonio, P. Gao et al., Diabetes mellitus, fasting glucose, and risk of cause-specific deaths. N. Engl. J. Med. 364, 829-841 (2011)

2. IDF Diabetes Atlas Update (2014), http://www.idf.org/diabete satlas/6e/Update2014

3. G.W.D. Landman, J.V. van Vliet-Ostaptchouk, N. Kleefstra, K.J.J. van Hateren, I. Drion, K.H. Groenier et al., Association between 9 p21 genetic variants and mortality risk in a prospective cohort of patients with type 2 diabetes (ZODIAC-15). Cardiovasc. Diabetol. 11, 138 (2012)

4. A.G.P. Sousa, G.F. Marquezine, P.A. Lemos, E. Martinez, N. Lopes, W.A. Hueb et al., TCF7L2 Polymorphism rs7903146 is associated with coronary artery disease severity and mortality. PLoS One 4, e7697 (2009)

5. D. Altshuler, J.N. Hirschhorn, M. Klannemark, C.M. Lindgren, M.C. Vohl, J. Nemesh et al., The common PPAR gamma Pro12Ala polymorphism is associated with decreased risk of type 2 diabetes. Nat. Genet. 26, 76-80 (2000)

6. J.M. Olefsky, Treatment of insulin resistance with peroxisome proliferator-activated receptor gamma agonists. J. Clin. Invest. 106, 467-472 (2000)

7. J.A. Dormandy, B. Charbonnel, D.J. Eckland, E. Erdmann, M. Massi-Benedetti, I.K. Moules et al., Secondary prevention of macrovascular events in patients with type 2 diabetes in the PROactive Study (PROspective pioglitAzone Clinical Trial In macroVascular Events): a randomised controlled trial. Lancet 366, 1279-1289 (2005)

8. A. Meirhaeghe, L. Fajas, N. Helbecque, D. Cottel, J. Auwerx, S.S. Deeb et al., Impact of the Peroxisome Proliferator Activated Receptor gamma2 Pro12Ala polymorphism on adiposity, lipids and non-insulin-dependent diabetes mellitus. Int. J. Obes. Relat. Metab. Disord. 24, 195-199 (2000)

9. A. Doney, B. Fischer, D. Frew, A. Cumming, D.M. Flavel, M. World et al., Haplotype analysis of the PPARgamma Pro12Ala and $\mathrm{C} 1431 \mathrm{~T}$ variants reveals opposing associations with body weight. BMC Genet. 3, 21 (2002)

10. C.J. Ostgren, U. Lindblad, O. Melander, A. Melander, L. Groop, L. Rastam, Peroxisome proliferator-activated receptor-gamma Pro12Ala polymorphism and the association with blood pressure in type 2 diabetes: skaraborg hypertension and diabetes project. J. Hypertens. 21, 1657-1662 (2003)

11. P.M. Ridker, N.R. Cook, S. Cheng, H.A. Elich, K. Lindpaintner, J. Plutzky et al., Alanine for proline substitution in the peroxisome proliferator-activated receptor gamma-2 (PPARG2) gene and the risk of incident myocardial infarction. Arterioscler. Thromb. Vasc. Biol. 23, 859-863 (2003)

12. A.S.F. Doney, B. Fischer, G. Leese, A.D. Morris, C.A.N. Palmer, Cardiovascular risk in type 2 diabetes is associated with variation at the PPARG locus a go-darts study. Arterioscler. Thromb. Vasc. Biol. 24, 2403-2407 (2004)

13. S. De Cosmo, N. Motterlini, S. Prudente, F. Pellegrini, R. Trevisan, A. Bossi et al., Impact of the PPARgamma2 Pro12Ala polymorphism and ACE inhibitor therapy on new-onset microalbuminuria in type 2 diabetes: evidence from BENEDICT. Diabetes 58, 2920-2929 (2009)

14. S. De Cosmo, S. Prudente, O. Lamacchia, E. Lapice, E. Morini, R. Di Paola et al., PPAR $\gamma 2$ P12A polymorphism and albuminuria in patients with type 2 diabetes: a meta-analysis of case-control studies. Nephrol. Dial. Transplant. 26, 4011-4016 (2011)

15. H. Zhang, S. Zhu, J. Chen, Y. Tang, H. Hu, V. Mohan et al., Peroxisome proliferator-activated receptor $\gamma$ polymorphism Pro12Ala Is associated with nephropathy in type 2 diabetes: evidence from meta-analysis of 18 studies. Diabetes Care 35, 1388-1393 (2012)

16. Y. RuiChao, B. Hong, H. SongMing, Association between the PPARG gene polymorphism and the risk of diabetic nephropathy: 
a meta-analysis. Genet. Test. Mol. Biomarkers 16, 429-434 (2012)

17. C.C. Szeto, K.M. Chow, P.Y.K. Poon, B.C.H. Kwan, P.K.Y. Li, Peroxisome proliferator-activated receptor-gamma gene polymorphism and risk of cardiovascular disease in patients with diabetic nephropathy. Am. J. Nephrol. 28, 715-722 (2008)

18. S. Bacci, S. Rizza, S. Prudente, B. Spoto, C. Powers, A. Facciorusso et al., The ENPP1 Q121 variant predicts major cardiovascular events in high-risk individuals evidence for interaction with obesity in diabetic patients. Diabetes 60, 1000-1007 (2011)

19. S. De Cosmo, M. Copetti, O. Lamacchia, A. Fontana, M. Massa, E. Morini et al., Development and validation of a predicting model of all-cause mortality in patients with type 2 diabetes mellitus. Diabetes Care 36, 2830-2835 (2013)

20. J.E. Wigginton, D.J. Cutler, G.R. Abecasis, A note on exact tests of Hardy-Weinberg equilibrium. Am. J. Hum. Genet. 76, 887-893 (2005)

21. S. Greenland, Quantitative methods in the review of epidemiologic literature. Epidemiol. Rev. 9, 1-30 (1987)

22. H.C. van Houwelingen, L.R. Arends, T. Stijnen, Advanced methods in meta-analysis: multivariate approach and meta-regression. Stat. Med. 21, 589-624 (2002)

23. J.A. Sterne, A.J. Sutton, J.P. Ioannidis, N. Terrin, D.R. Jones et al., Recommendations for examining and interpreting funnel plot asymmetry in meta-analyses of randomised controlled trials. BMJ 343, d4002 (2011)

24. J.P. Ioannidis, T.A. Trikalinos, The appropriateness of asymmetry tests for publication bias in meta-analyses: a large survey. CMAJ 176, 1091-1096 (2007)

25. O. Ludovico, F. Pellegrini, R. Di Paola, M. Minenna, S. Mastroianno, M. Cardellini et al., Heterogeneous effect of peroxisome proliferator-activated receptor 2 Ala12 variant on type 2 diabetes risk. Obesity 15, 1076-1081 (2007)

26. U. Vogel, S. Segel, C. Dethlefsen, A. Tjønneland, A. Thoustrup Saber, H. Wallin et al., PPAR $\gamma$ Pro12Ala polymorphism and risk of acute coronary syndrome in a prospective study of Danes. BMC Med. Genet. 10, 52 (2009)

27. M. Barbieri, M. Bonafé, M.R. Rizzo, E. Ragno, F. Olivieri, F. Marchegiani et al., Gender specific association of genetic variation in peroxisome proliferator-activated receptor (PPAR)g-2 with longevity. Exp. Gerontol. 39, 1095-1100 (2004)

28. M.R. Corbo, A. Pinto, R. Scacchi, Gender-specific association between FSHR and PPARG common variants and human longevity. Rejuvenation Research 16, 21-27 (2013)

29. E. Morini, V. Tassi, D. Capponi, O. Ludovico, B. Dallapiccola, V. Trischitta et al., Interaction between $P P A R \gamma 2$ variants and gender on the modulation of body weight. Obesity 16, 1467-1470 (2008)

30. L. Marullo, J.S. El-Sayed Moustafa, I. Prokopenko, Insights into the genetic susceptibility to type 2 diabetes from genome-wide association studies of glycaemic traits. Curr. Diab. Rep. 14, 551 (2014)

31. S. Bacci, S. Prudente, M. Copetti, B. Spoto, S. Rizza, R. Baratta et al., Joint effect of insulin signaling genes on cardiovascular events and on whole body and endothelial insulin resistance. Atherosclerosis 226, 140-145 (2013)

32. S. Prudente, E. Morini, L. Marselli, R. Baratta, M. Copetti, C. Mendonca et al., Joint effect of insulin signaling genes on insulin secretion and glucose homeostasis. J. Clin. Endocrinol. Metab. 98, E1143-E1147 (2013)

33. C. Menzaghi, A. Fontana, M. Copetti, S. Rizza, B. Spoto, P. Buranasupkajorn et al., Joint effect of insulin signaling genes on all-cause mortality. Atherosclerosis 237, 639-644 (2014)
34. E.S. Ford, Risks for all-cause mortality, cardiovascular disease, and diabetes associated with the metabolic syndrome: a summary of the evidence. Diabetes Care 28, 1769e1778 (2005)

35. K.J. Ausk, E.J. Boyko, G.N. Ioannou, Insulin resistance predicts mortality in nondiabetic individuals in the U.S. Diabetes Care 33, $1179 \mathrm{e} 1185$ (2010)

36. S. Prudente, H. Shah, D. Bailetti, M. Pezzolesi, P. Buranasupkajorn, L. Mercuri et al., Genetic variant at the GLUL locus predicts all-cause mortality in patients with type 2 diabetes. Diabetes 64, 2658-2663 (2015)

37. C. Menzaghi, M. Xu, L. Salvemini, C. De Bonis, G. Palladino, T. Huang et al., Circulating adiponectin and cardiovascular mortality in patients with type 2 diabetes mellitus: evidence of sexual dimorphism. Cardiovasc. Diabetol. 13, 130 (2014)

38. R. Di Paola, A. Marucci, A. Fontana, C. Menzaghi, L. Salvemini, M. Copetti et al., Role of obesity on all-cause mortality in whites with type 2 diabetes from Italy. Acta Diabetol. 50, 971-976 (2013)

39. C. Menzaghi, S. Bacci, L. Salvemini, C. Mendonca, G. Palladino, A. Fontana et al., Serum resistin, cardiovascular disease and allcause mortality in patients with type 2 diabetes. PLoS One 8 , e64729 (2013)

40. S. De Cosmo, O. Lamacchia, A. Pacilli, S. Fariello, S. Pinnelli, A. Fontana et al., Normoalbuminuric renal impairment and all-cause mortality in type 2 diabetes mellitus. Acta Diabetol. 51, 687-689 (2014)

41. A. Fontana, S. Spadaro, M. Copetti, B. Spoto, L. Salvemini, P. Pizzini et al., Association between resistin levels and all-cause and cardiovascular mortality: a new study and a systematic review and meta-analysis. PLoS One 10, e0120419 (2015)

42. A. Pacilli, O. Lamacchia, A. Fontana, M. Copetti, M. Cignarelli, V. Trischitta et al., Target values of cardiovascular risk factors are not associated with all-cause mortality in patients with type 2 diabetes mellitus. PLoS One 10, e0124536 (2015)

43. T.M. Morgan, L. Xiao, P. Lyons, B. Bethany Kassebaum, H.M. Krumholz, J.A. Spertus, Investigation of 89 candidate gene variants for effects on all-cause mortality following acute coronary syndrome. BMC Med. Genet. 9, 66 (2008)

44. L. Gallicchio, H.H. Chang, D.K. Christo, L. Thuita, H.Y. Huang, P. Strickland et al., Single nucleotide polymorphisms in obesityrelated genes and all-cause and cause-specific mortality: a prospective cohort study. BMC Med. Genet. 10, 103 (2009)

45. A. Jorsal, L. Tarnow, M. Lajer, J. Ek, T. Hansen, O. Pedersen et al., The PPAR 2 Pro12Ala variant predicts ESRD and mortality in patients with type 1 diabetes and diabetic nephropathy. Mol. Genet. Metab. 94, 347-351 (2008)

46. C.T. Chao, Y.C. Chen, C.K. Chiang, J.W. Huang, F.C. Hu, C.C. Fang et al., Sequence variants of peroxisome proliferator-activated receptor-gamma gene and the clinical courses of patients with end-stage renal disease. Dis. Markers 2015, 763459 (2015)

47. Q. Yao, L. Nordfors, J. Axelsson, O. Heimbürger, A.R. Qureshi, P. Barany et al., Peroxisome proliferator-activated receptor gamma polymorphisms affect systemic inflammation and survival in end-stage renal disease patients starting renal replacement therapy. Atherosclerosis 182, 105-111 (2005)

48. J.J. Regieli, J.W. Jukema, P.A. Doevendans, A.H. Zwinderman, Y. van der Graaf, J.J. Kastelein et al., $P P A R \gamma$ variant influences angiographic outcome and 10-year cardiovascular risk in male symptomatic coronary artery disease patients. Diabetes Care 32, 839-844 (2009) 